\title{
Computer-Aided Analysis of Flow in Water Pipe Networks after a Seismic Event
}

\author{
Won-Hee Kang, ${ }^{1}$ Young-Joo Lee, ${ }^{2}$ and Chunwei Zhang ${ }^{1}$ \\ ${ }^{1}$ Centre for Infrastructure Engineering, Western Sydney University, Penrith, NSW 2751, Australia \\ ${ }^{2}$ School of Urban and Environmental Engineering, Ulsan National Institute of Science and Technology (UNIST), Ulsan 44919, \\ Republic of Korea \\ Correspondence should be addressed to Young-Joo Lee; ylee@unist.ac.kr
}

Received 28 December 2016; Accepted 20 February 2017; Published 8 March 2017

Academic Editor: Anders Eriksson

Copyright (c) 2017 Won-Hee Kang et al. This is an open access article distributed under the Creative Commons Attribution License, which permits unrestricted use, distribution, and reproduction in any medium, provided the original work is properly cited.

\begin{abstract}
This paper proposes a framework for a reliability-based flow analysis for a water pipe network after an earthquake. For the first part of the framework, we propose to use a modeling procedure for multiple leaks and breaks in the water pipe segments of a network that has been damaged by an earthquake. For the second part, we propose an efficient system-level probabilistic flow analysis process that integrates the matrix-based system reliability (MSR) formulation and the branch-and-bound method. This process probabilistically predicts flow quantities by considering system-level damage scenarios consisting of combinations of leaks and breaks in network pipes and significantly reduces the computational cost by sequentially prioritizing the system states according to their likelihoods and by using the branch-and-bound method to select their partial sets. The proposed framework is illustrated and demonstrated by examining two example water pipe networks that have been subjected to a seismic event. These two examples consist of 11 and 20 pipe segments, respectively, and are computationally modeled considering their available topological, material, and mechanical properties. Considering different earthquake scenarios and the resulting multiple leaks and breaks in the water pipe segments, the water flows in the segments are estimated in a computationally efficient manner.
\end{abstract}

\section{Introduction}

Water pipe networks constitute one of the largest of our essential infrastructure assets and make a substantial contribution to economic services, industrial activities, quality of life, and the environment. Their primary duty is to provide ample amounts of water at a pressure that is sufficient to satisfy the demands of all consumers. A reliable water supply is essential to communities and is one of the so-called "services of general interest" that are vital to general welfare, public health, and the collective security of populations, as well as economic activities [1]. If a natural or man-made disaster occurs, however, water pipe networks are often vulnerable to structural failures and may lose a significant amount of water or even become nonoperational due to leakage from and breakage of the pipe segments. This prevents them from carrying the specified quantities of water at the required pressure heads, and any postdisaster failure of the water supply obviously causes considerable public inconvenience.
In this context, it is imperative to be able to predict the postdisaster flow in a water network, considering possible leaks and breaks to enable prompt risk-informed decisionmaking on hazard mitigation and disaster management.

Many research efforts have attempted to estimate the seismic performance or probabilistic flow quantity of water supply networks using Monte Carlo simulation (MCS) or nonsimulation-based methods. Recently, several researchers [26] studied a water network in Memphis, Tennessee, USA, which is interconnected with the area's power network. They estimated the seismic performance of the network through the application of connectivity analysis or performance measures while considering cascading failure scenarios. They used various methods including MCS and proposed the use of non-simulation-based methods. Other researchers $[7,8]$ studied a network in the city of Limassol, Cyprus, and conducted a seismic connectivity analysis using a semianalytical path enumeration method and MCS. However, all of these studies focused on connectivity-based performance 
estimation and did not consider actual quantities such as the head pressures or pipe flows because the connectivity analysis is complicated by the necessity to consider the complex network topology. There have been only a few studies of the seismic performance of a network that have also considered flow quantities. Zolfaghari and Niari [9] studied a network in northwestern Tehran, where they considered a total of 30 damage scenarios and the resulting water flows, consisting of combinations of five damage states and six wasted water states for the system's water tanks. They provided simple analytical solutions for the water disruption probabilities based on a Poisson process. Lee et al. [10] studied a benchmark transportation network in Sioux Falls, USA, considering ten seismically vulnerable reinforced-concrete bridges and four possible damage states. Although they did not address the water network, they derived formulations for considering the quantities resulting from combinations of multiple component damage states within a network. The quantities were simply considered from the maximum capacities of the transportation network. Torii and Lopez [11] worked on three small numerical network models under nonnatural-hazard conditions and then evaluated the reliability of each component based on nodal head expectations without considering combinations of the component damage states. Uncertainties in the boundary conditions and pipe roughness were considered, and the nodal heads were calculated using a gradient-based method. The integrated use of an adaptive response surface approach with FORM (First-Order Reliability Method) analysis was proposed. Liu et al. [12] extensively applied the GIRAFFE software [13], originally designed for the hydraulic analysis of water networks that had been damaged by the 1994 Northridge earthquake, to water supply networks in Taiwan. MCS analyses were used to determine serviceability indices for demand requirements in several areas. Yoo et al. [14] proposed an optimal design scheme for a water distribution system to maximize the seismic reliability estimated based on a proposed indicator. However, these studies are still not able to consider network quantities along with all possible seismic hazard scenarios that would cause leaks and breaks in multiple pipe segments. Further study is consequently needed to attain more realistic seismic performance predictions for water pipe networks that take network quantities into account.

This paper proposes a framework for a system-level probabilistic water network flow analysis after a seismic event. The first part of the proposed framework involves the computational modeling of a water pipe network considering the components that are leaking and broken components due to earthquake shaking. In this study, multiple software packages including ArcGIS [15], EPANET [16], and GIRAFFE [13] are integrated, and they can be flexibly further improved as needed. The second part of the proposed framework is a computationally efficient analysis process for the probabilistic postearthquake water flow analysis of a network considering system-level damage scenarios such as the leakage from and breakage of multiple pipes using the matrix-based system reliability (MSR) analysis framework $[10,17]$ and the branchand-bound method $[18,19]$. The branch-and-bound method sequentially identifies critical damage scenarios according to their likelihoods, and reduces the size of the vectors/matrices in the MSR method by considering only the most important scenarios. Preliminary results on the proposed algorithm were reported in [20].

\section{Proposed Framework}

The proposed framework includes a computational network modeling procedure for hydraulic analysis, a damage modeling procedure for leaks from and breaks in pipe segments, and a system-level probabilistic flow analysis procedure combining the MSR method and the branch-and-bound method. The modeling part of the framework can be further improved by introducing more realistic assumptions and precise modeling schemes.

2.1. Introduction. The first procedure of the proposed framework consists of network modeling for hydraulic analysis, which includes the following steps. (i) The topology of the water pipe network of interest is prepared. An initial drawing of the model is overlaid on a base map obtained from Google Earth, originally developed by Keyhole Inc. in 2001 and later acquired by Google in 2004. Using Google Earth, the locations of the junctions and the pipes of the network are roughly determined. The other parameters, including the pipe diameter, roughness, and the locations of pumps, reservoirs, and tanks, are not included in the model at this stage. The model is stored as a keyhole markup language (KML) file containing the network's coordinate information for pipelines and junctions. (ii) The KML information from Google Earth is decompressed using GPS Visualizer, an Internet-based public-domain software product developed in 2002. Through the use of GPS Visualizer, the coordinates are extracted from the Google Earth KML file. A new KML file is created using GPS Visualizer which contains the accurate coordinates and ground profile of the water pipeline network. (iii) The KML file created in GPS Visualizer is imported into the ArcGIS software to undertake further modeling of the water pipeline network. ArcGIS is a software product based on the Geographic Information System (GIS) and developed by ESRI. In ArcGIS, additional parameter information such as the pipe diameter, pipe roughness, elevation of the junctions, and water demand locations and levels is added. Graphically unsnapped junctions in the Google Earth model are fully snapped using the snapping tool in ArcGIS with 0.001 decimal degrees, for example. A drawing interchange format (DXF) file is created in ArcGIS and then imported into EPANET (a hydraulic analysis software product developed by the US Environmental Protection Agency). Since EPANET cannot read DXF files directly, EPACAD is used to convert the DXF file into an input file for EPANET. (iv) In EPANET, the remaining parameters are defined, including the locations of reservoirs, tanks, and pumps, the required demand at junctions, and other flowrelated parameters. Once the network modeling is complete, a hydraulic analysis can be carried out with EPANET to estimate the water flows in the pipeline segments and the head 


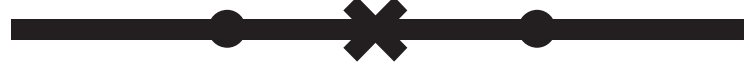

Pipe break

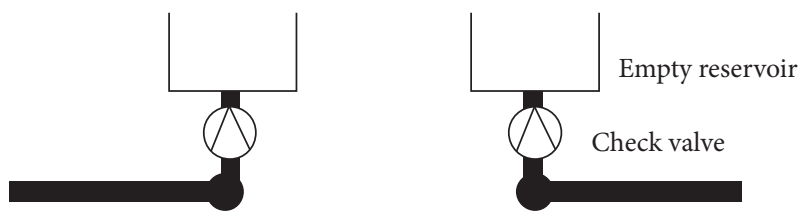

FIgURE 1: Hydraulic model of pipe break.

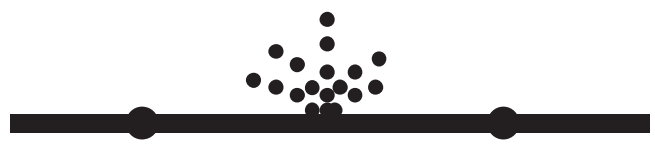

Pipe leak

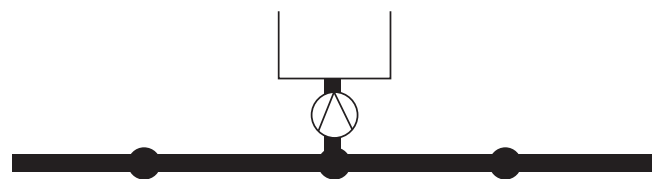

Figure 2: Hydraulic model of pipe leak.

pressures of the pipe junctions. EPANET uses a gradientbased algorithm developed by Todlini and Pilati [23] for network static analysis, in which the head losses along the pipes are computed using the Hazen-Williams formula.

2.2. System Damage Modeling for Multiple Pipe Leaks and Breaks. To model leaks and breaks in multiple pipe segments after a seismic event, a modeling scheme in GIRAFFE [13] was adopted and simplified such that pipe segment damage can assume only two states, namely, leaks or breaks, to simplify the computation. The modeling details for pipe breaks and leaks are illustrated in Figures 1 and 2, respectively. Figure 1 shows the modeling of a pipe break by removing the corresponding pipe element. The corresponding water discharge is modeled by installing two empty reservoirs at the two end-points of the removed element. To model water discharge from the broken pipe into the atmosphere, one-way check valves are installed.

Figure 2 shows the modeling of a pipe leak, where an empty reservoir is installed at the mid-point of the leaked pipe, and the leaking pipe and reservoir are connected through a fictitious pipe and a one-way check valve. The diameter of the fictitious pipe determines the leak rate and, in this study, it was assumed to be $1 / 6$ that of the original pipe. All of these modeling procedures are automated in MATLAB ${ }^{\circledR}$ modifying the EPANET MATLAB Toolkit [24] to model random breakages of and leaks from multiple pipelines after an earthquake. For better modeling of leakages and breakages, alternative techniques such as assigning emitter discharge coefficients may also be used.

2.3. MSR-Based Posthazard Flow Analysis. In the proposed framework, the MSR-based uncertainty quantification method [10] was employed to carry out a posthazard water pipeline network flow analysis, in which the automated modeling procedure introduced in Sections 2.1 and 2.2 is used to estimate the water flow quantities. Consider a water pipe network system consisting of $n$ pipeline components. The $i$ th component has $d_{i}$ prescribed damage states, $i=1, \ldots, n$. Thus, the system has a total of $d_{1} \times d_{2} \times \cdots \times d_{n}$ system states as determined by the component damage states. In this study, every $d_{i}$ is assumed to be 3 , corresponding to there being no damage, leaks, or breaks, such that the system has $3^{n}$ damage states, but this can be further generalized for other applications. Let $P_{i,(j)}, i=1, \ldots, n, j=1, \ldots, d_{i}$, denote the probability of the $i$ th component in the $j$ th damage state. By assuming that all the components are statistically independent, the probability of each system state can be obtained as the product of the corresponding component probabilities; that is,

$$
\mathbf{p}=\left[\begin{array}{c}
P_{(1,1, \ldots, 1)} \\
P_{(2,1, \ldots, 1)} \\
\vdots \\
P_{\left(d_{1}, d_{2}, \ldots, d_{n}\right)}
\end{array}\right]=\left[\begin{array}{c}
P_{1,(1)} \times P_{2,(1)} \times \cdots \times P_{n,(1)} \\
P_{1,(2)} \times P_{2,(1)} \times \cdots \times P_{n,(1)} \\
\vdots \\
P_{1,\left(d_{1}\right)} \times P_{2,\left(d_{2}\right)} \times \cdots \times P_{n,\left(d_{n}\right)}
\end{array}\right],
$$

where $\mathbf{p}$ is the probability vector showing all possible system states and $P_{(\ldots)}$ with multiple numbers in the subscript denotes the probability of a system damage state. The numbers in the subscript mean the component damage states that determine the system state. For example, $P_{(2,1, \ldots, 1)}$ in the second row indicates that all of the components are in the first damage state except for the first, which is in the second damage state.

For each system state, any corresponding system quantity such as the posthazard flow of each pipeline can be estimated by using the water flow analysis model proposed in Sections 2.1 and 2.2. For each of the system states in (1), the system quantities are evaluated as follows:

$$
\mathbf{q}=\left[\begin{array}{c}
Q_{(1,1, \ldots, 1)} \\
Q_{(2,1, \ldots, 1)} \\
\vdots \\
Q_{\left(d_{1}, d_{2}, \ldots, d_{n}\right)}
\end{array}\right]=\left[\begin{array}{c}
f\left(q_{1,(1)}, q_{2,(1)}, \ldots, q_{n,(1)}\right) \\
f\left(q_{1,(2)}, q_{2,(1)}, \ldots, q_{n,(1)}\right) \\
\vdots \\
f\left(q_{1,\left(d_{1}\right)}, q_{2,\left(d_{2}\right)}, \ldots, q_{n,\left(d_{n}\right)}\right)
\end{array}\right]
$$

where $\mathbf{q}$ is the quantity vector, $Q_{(\ldots)}$ denotes the system quantity of the system state as determined by the component damage state given by the subscript, and $f(\cdot)$ denotes the posthazard flow analysis model proposed in Sections 2.1 and 2.2 .

Using the probability vector $\mathbf{p}$ in (1) and the quantity factor $\mathbf{q}$ in (2), the statistical parameters and the probability functions of the system quantities, such as the mean, the variance, and the cumulative distribution function (CDF) of 
the system quantity, can easily be estimated by the following matrix calculations [10]:

$$
\begin{aligned}
\mu_{\mathrm{Q}} & =\mathbf{q}^{\mathrm{T}} \mathbf{p}, \\
\sigma_{\mathrm{Q}}^{2} & =\mathbf{p}^{\mathrm{T}}(\mathbf{q} \cdot * \mathbf{q})-\mu_{\mathrm{Q}}^{2}, \\
F_{\mathrm{Q}}(q) & =P(\mathrm{Q} \leq q)=\sum_{\forall k: q_{k} \leq q} p_{k},
\end{aligned}
$$

where ".*" denotes the element-wise product of two vectors and $p_{i}$ and $q_{i}$ are the $k$ th elements of vectors $\mathbf{p}$ and $\mathbf{q}$, respectively.

\subsection{Branch-and-Bound Method for Efficient Probabilistic} Flow Analysis. The system-level probabilistic flow analysis described in Section 2.3 may incur a huge computational cost in the case of a large system. This is because the number of system states (i.e., $d_{1} \times d_{2} \times \cdots \times d_{n}$ ) increases exponentially with the number of components. In this study, we propose an efficient method based on the branch-and-bound method $[18,19]$.

The branch-and-bound method is used to sort the system state probability vector in (1), as follows. We start from the probability vector of the first component only, that is, $\left[P_{1(1)}, \ldots, P_{1\left(d_{1}\right)}\right]^{\mathrm{T}}$. We find the element with the maximum value in this vector and multiply it by the probability vector of the next component, $\left[P_{2(1)}, \ldots, P_{2\left(d_{2}\right)}\right]^{\mathrm{T}}$. Then, the total size of the probability vector increases to $\left(d_{1}+d_{2}-1\right)$. Next, we repeat the finding of the element with the maximum value in this larger-size vector and check if this element was branched out in the previous process. If so, we multiply it by the vector of the next component, $\left[P_{3(1)}, \ldots, P_{3\left(d_{3}\right)}\right]^{\mathrm{T}}$, to increase the vector size to $\left(d_{1}+d_{2}+d_{3}-2\right)$. If not, we multiply the element by the vector of the second component, $\left[P_{2(1)}, \ldots, P_{2\left(d_{2}\right)}\right]^{\mathrm{T}}$, to increase the vector size to $\left(d_{1}+2 d_{2}-2\right)$. These processes are repeated to prioritize the important system states and evaluate their probabilities.

For each process, we check whether the element with the maximum value contains all $n$ network components in the product. If so, the maximum value and the corresponding system state and flow quantity are stored, and the element having the second-highest system probability is branched out to continue the process. Critical system states and their probabilities are collected until the sum of the system state probabilities reaches a value close to either 1.0 or a target value. Note that the size of this partial probability vector is significantly smaller than the full vector in (1) because many of the system states have negligible likelihoods. Then, a normalizing process that divides all of the stored values by their sum is conducted so that the sum of the probabilities in the partial vector becomes 1.0. By replacing the complete probability vector in (1) by the normalized partial probability vector, we can perform a probabilistic flow analysis with significantly reduced computer memory requirements and improved efficiency.

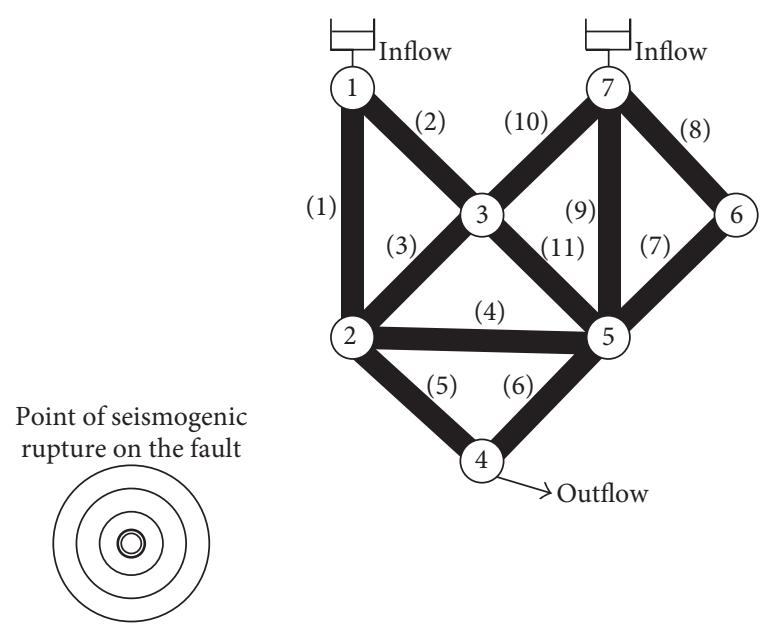

FIGURE 3: Example water pipe network with 11 pipe segments.

\section{Applications}

3.1. 11-Pipe Water Supply Network. The proposed framework is applied to the water pipe network shown in Figure 3, which is modeled based on a network example given by Pudar and Liggett [25] and Vítkovský et al. [26]. Since the model is not based on a specific geological area, the base map in Google Earth is chosen arbitrarily but the other modeling procedures proposed in this paper are used as is. It has 11 pipes (indexed by the numbers in parentheses), seven nodes (indexed by the numbers in circles), inflows from two reservoirs, and one outflow at a demand node (node 4). A constant demand at a fixed-time point is assumed with a specified demand value. The diameter of every pipe is $254 \mathrm{~mm}$, and the lengths are $1372 \mathrm{~m}, 762 \mathrm{~m}, 762 \mathrm{~m}, 1067 \mathrm{~m}, 762 \mathrm{~m}, 762 \mathrm{~m}, 762 \mathrm{~m}, 914 \mathrm{~m}$, $1219 \mathrm{~m}, 762 \mathrm{~m}$, and $762 \mathrm{~m}$. These lengths are provided in the order of the pipe numbers from 1 to 11 . The Hazen-Williams coefficient is set to $W=120$.

If an earthquake occurs, it is assumed that each pipeline in the network will assume one of the following three damage states: undamaged, leaking, or broken. The failure probabilities of the pipes are estimated using the equation below for repair rates, given as a function of the peak ground velocity (PGV) in the HAZUS technical manual [27], which is defined by the average number of failures per unit length $(\mathrm{km})$ of a pipe. This equation is based on a fitting to the empirical pipeline damage data presented in a work done by O'Rourke and Ayala [28]. For better accuracy, this equation may be replaced with alternative equations such as the equation proposed by Isoyama et al. [29] considering further correction factors regarding pipe and soil characteristics.

$$
\text { repair rate } \cong 0.0001 \times(\mathrm{PGV})^{2.25} \text {. }
$$

The failure probability of each pipe is computed using a Poisson process along a dimension of length. This paper deals only with failures induced by ground shaking and uses the repair rate model given by (4); ground failures are ignored. 
The PGV is computed from the following attenuation relationship [30]:

$$
\begin{aligned}
\ln (\mathrm{PGV})= & \ln (\mathrm{PGA})+0.26+0.29 \mathrm{M} \\
& -1.44 \ln [r+0.0203 \exp (0.958 M)] \\
& +1.89 \ln [r+0.361 \exp (0.576 \mathrm{M})] \\
& +(0.0001-0.000565 M) r-0.12 F \\
& -0.15 S_{S R}-0.30 S_{S R} \\
& -0.75 \tanh (0.51 D)\left(1-S_{H R}\right)-f_{V}(D),
\end{aligned}
$$

where

$$
\begin{aligned}
\ln (\mathrm{PGA}) \\
=-3.512+0.904 M \\
\quad-1.328 \ln \left\{\sqrt{r^{2}+[0.149 \exp (0.647 M)]^{2}}\right\} \\
+[1.125-0.112 \ln r-0.0957 M] F \\
+[0.440-0.171 \ln r] S_{S R} \\
+[0.405-0.222 \ln r] S_{H R},
\end{aligned}
$$

where PGA is the peak ground acceleration, $M$ denotes the earthquake magnitude (assumed to vary between 6.5 and 8.0), $F$ represents the fault type, assumed to be 0 for strikeslip type faults, $S_{S R}$ and $S_{H R}$ define the local site conditions, assumed to be alluvium or firm soil $S_{S R}=S_{H R}=0, D$ denotes the distance to the bedrock, assumed to be $0.45 \mathrm{~km}$, and $r$ is the distance between the center of each pipe and the epicenter. The distances between the pipes and the epicenter are $3.9 \mathrm{~km}, 4.2 \mathrm{~km}, 3.8 \mathrm{~km}, 3.9 \mathrm{~km}, 3.5 \mathrm{~km}, 4.0 \mathrm{~km}, 4.1 \mathrm{~km}$, $4.7 \mathrm{~km}, 4.9 \mathrm{~km}, 4.5 \mathrm{~km}$, and $4.5 \mathrm{~km}$ (from pipes 1 to 11 ), respectively. For $D<1 \mathrm{~km}, f_{V}(D)$ is given as

$$
f_{V}(D)=-0.30\left(1-S_{H R}\right)(1-D)-0.15(1-D) S_{S R} .
$$

When a pipe segment is damaged by the ground shaking of an earthquake, it can assume either one of two states: leaking or broken. The likelihoods of these occurring are assumed to be 0.8 and 0.2 , according to the probability distribution used in Zolfaghari and Niari [9]. Then, the probabilities of the leakage and breakage of a pipe in an earthquake are computed as the product of the failure probability obtained by the use of the repair rate in (4) and the probabilities of the occurrence of either a leak or break in the event of an earthquake. The undamaged state is calculated as one minus the probability of failure (leak or break) of the pipe.

Using the proposed flow-based reliability analysis framework, the mean, standard deviation, and coefficient of variation (c.o.v.) of the flow rate in pipe 5 for given earthquake magnitudes were estimated. These are listed in Table 1. In this table, earthquake magnitudes of 6.0, 7.0, and 8.5 are selected, while an unknown earthquake magnitude is considered by using the probability density function (PDF) of the earthquake magnitude used by Kang et al. [31]. From
TABle 1: Mean, standard deviation, and c.o.v. of water flow from node 1 to pipe 5 .

\begin{tabular}{lccc}
\hline & Mean $\left(\mathrm{m}^{3} /\right.$ day $)$ & Standard deviation $\left(\mathrm{m}^{3} /\right.$ day $)$ & c.o.v. \\
\hline$M=6.0$ & 8219.4 & 11736 & 1.4278 \\
$M=7.0$ & 9164 & 17396 & 1.8983 \\
$M=8.0$ & 9182.7 & 23725 & 2.5837 \\
Uncertain $M$ & 8934.4 & 16461 & 1.8335 \\
\hline
\end{tabular}

TABLE 2: Mean, standard deviation, and c.o.v. of water flow in pipe 6.

\begin{tabular}{lccc}
\hline & Mean $\left(\mathrm{m}^{3} /\right.$ day $)$ & Standard deviation $\left(\mathrm{m}^{3} /\right.$ day $)$ & c.o.v. \\
\hline$M=6.0$ & -3746 & 8224.4 & 2.1955 \\
$M=7.0$ & -2907.3 & 14166 & 4.8725 \\
$M=8.0$ & -2654.3 & 20539 & 7.738 \\
Uncertain $M$ & -3100.8 & 13107 & 4.3982 \\
\hline
\end{tabular}

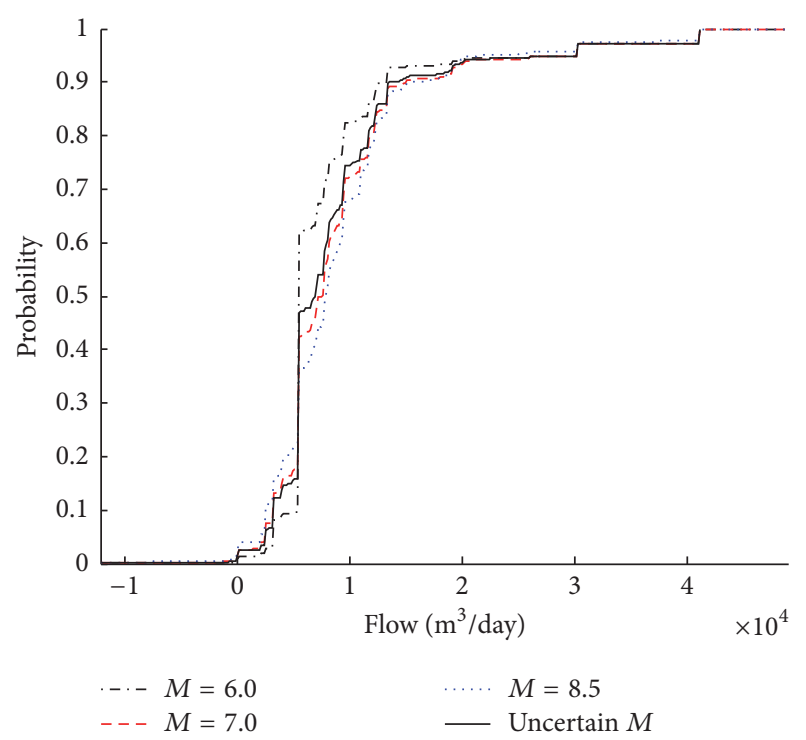

Figure 4: Probability of water flow in pipe 5.

the results, it can be seen that the flow in pipe 5 increases with the earthquake magnitude. This is because the pipes in the network are more likely to be damaged as the earthquake magnitude increases and thus the chance of water loss will increase, such that the flow in pipe 5 should increase to compensate for the water loss and maintain the required outflow demand at node 4 . Figure 4 plots the probability of the water flow.

The results for pipe 6 are listed in Table 2. All the mean values are negative, which implies that the assumed direction of flow is opposite to that of the actual flow. As the earthquake magnitude increases, the mean flow rates decrease due to water loss caused by leaks and breaks. Note that if we add the absolute mean flow rates for pipes 5 and 6 , the resulting value is almost constant due to the demand flow at node 4 , for all of the considered earthquake magnitudes. This means that even if the flow rates of pipes 5 and 6 change, their difference is 


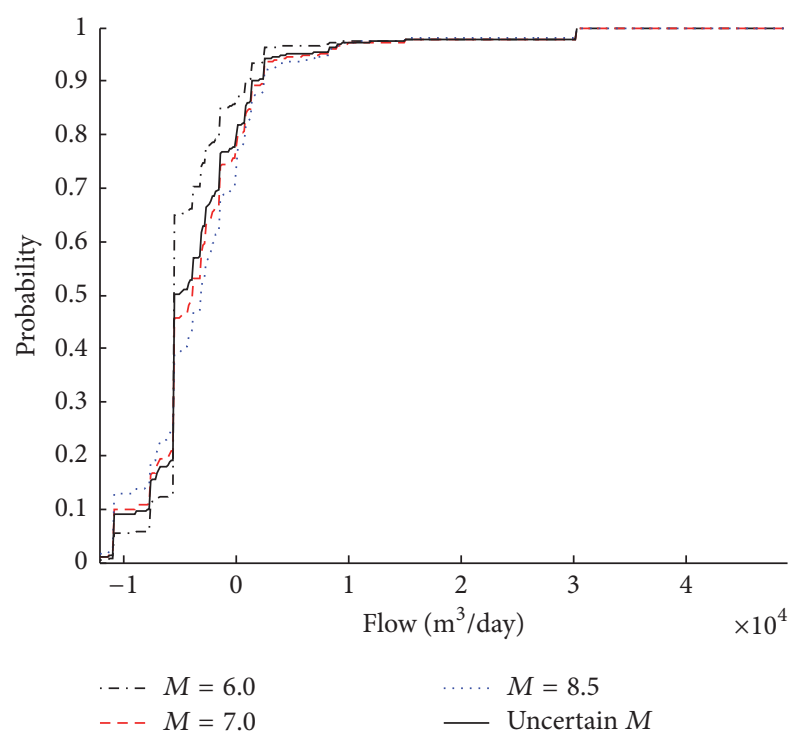

Figure 5: Probability of water flow in pipe 6.

always the same as the required outflow demand at node 4 . Figure 5 plots the probability of the water flow in pipe 6 .

The computational cost incurred for the analysis of the full system states is about 148 hours using MATLAB on a computer with an Intel I7 CPU $(2.80 \mathrm{GHz})$ and $3 \mathrm{~GB}$ of RAM, and it can be considered as unaffordable. However, it should be noted that, for all the results and plots, the quantity vector q in (2) was evaluated once only, and the change in the earthquake magnitudes and the corresponding changes in the pipe damage probabilities were handled only by updating the probability vector in (1), which significantly reduces the computational cost, as the greatest computational effort is incurred for the evaluation of the quantity vector $\mathbf{q}$, which requires repeated evaluation of the flow analyses.

To further reduce the computational cost, the branchand-bound method introduced in Section 2.4 is employed. The mean flow rates of the pipes are calculated as shown in Figures 6-9 for an earthquake magnitude of $M=7.0$. The analysis is repeated for an increasing number of branches, using the branch-and-bound method and represented by the sum of the probabilities in the probability vector, $\mathbf{p}$. The results are represented as two cases, such that the vector $\mathbf{p}$ is normalized by the sum of the probabilities and the vector $\mathbf{p}$ is not normalized.

Figure 6 compares the results obtained with the proposed branch-and-bound approach and the Monte Carlo simulations when the sum of the probabilities of the identified full branches has not reached 1 . The line of black solid circles represents the results obtained with the branch-and-bound approach when the results have been normalized by the sum of the probabilities; the dotted line of stars represents the results obtained with MCS. In addition, the line of empty circles represents the branch-and-bound approach results when the results have not been normalized by the sum of the probabilities. The analyses are repeated for a sum of probabilities varying from 0.1 to 0.99 . When the sum of

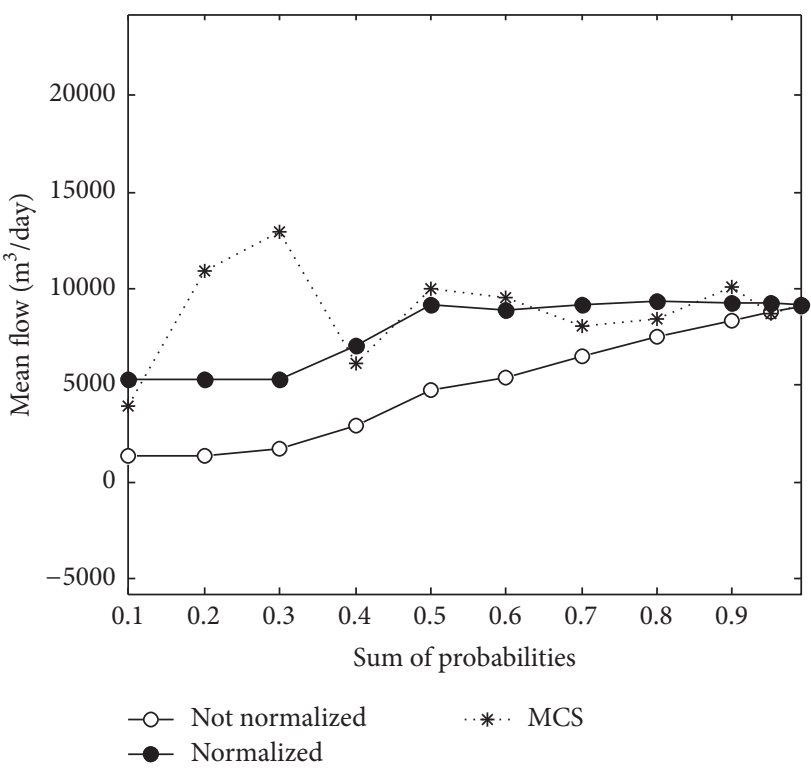

FIGURE 6: Comparison of results obtained with proposed branchand-bound approach and by Monte Carlo simulation for pipe 5 .

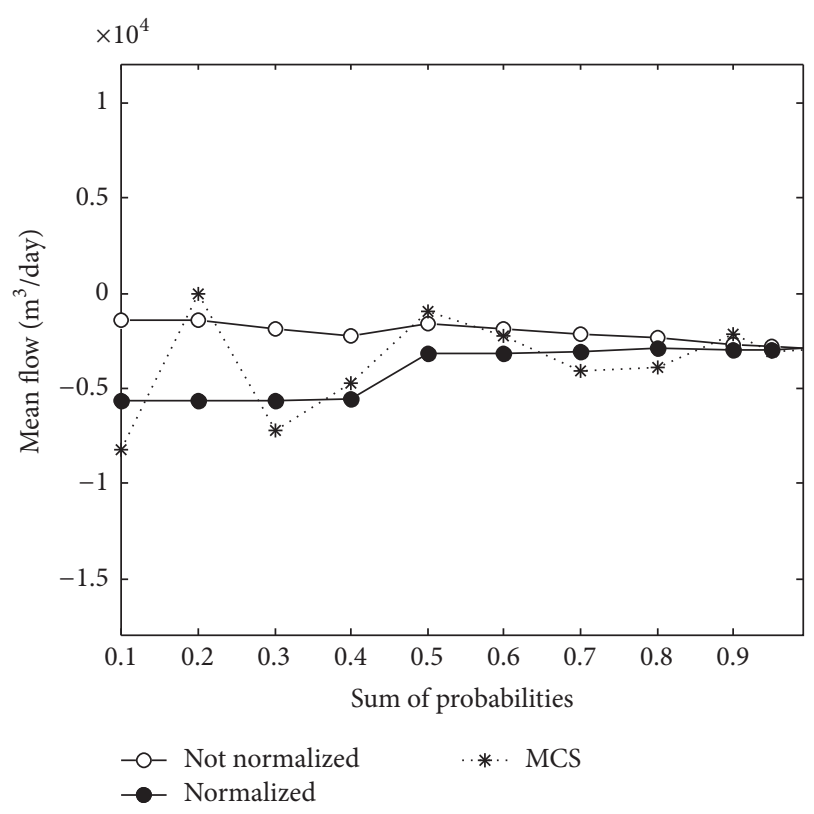

FIGURE 7: Comparison of results obtained with proposed branchand-bound approach and by Monte Carlo simulation for pipe 6 .

probabilities is between 0.1 and 0.9 , the interval is set to 0.1 , and then two more points at 0.95 and 0.99 are additionally considered. To make a fair comparison, MCS is carried out for exactly the same computation time as for the branch-andbound analysis.

In Figure 6, as the sum of the probabilities increases, the normalized and nonnormalized results obtained using the branch-and-bound method converge to a value of 


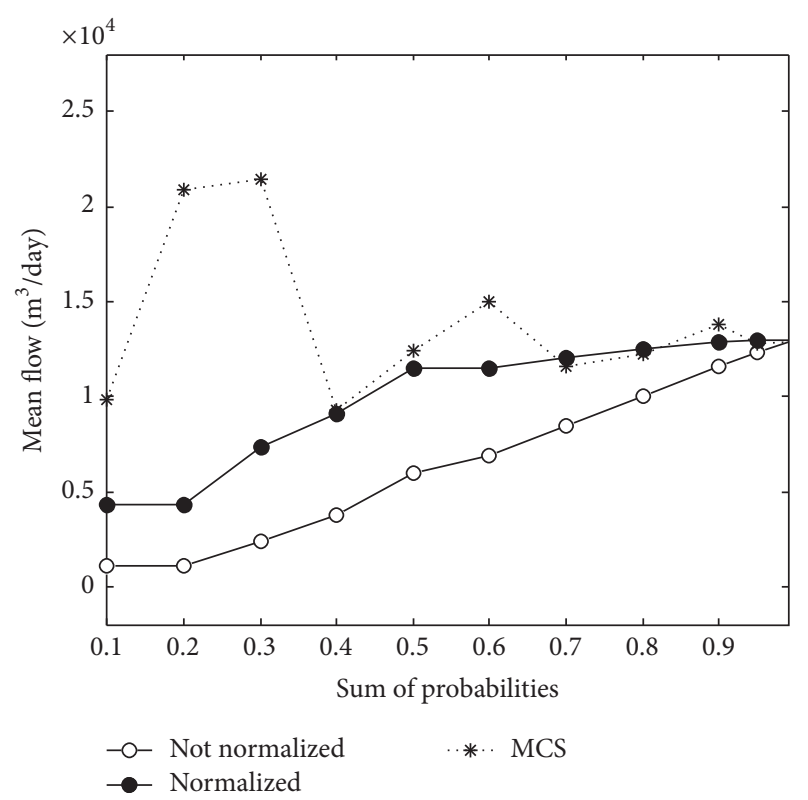

FIgURE 8: Comparison of the results obtained with proposed branch-and-bound approach and by Monte Carlo simulation for pipe 1 .

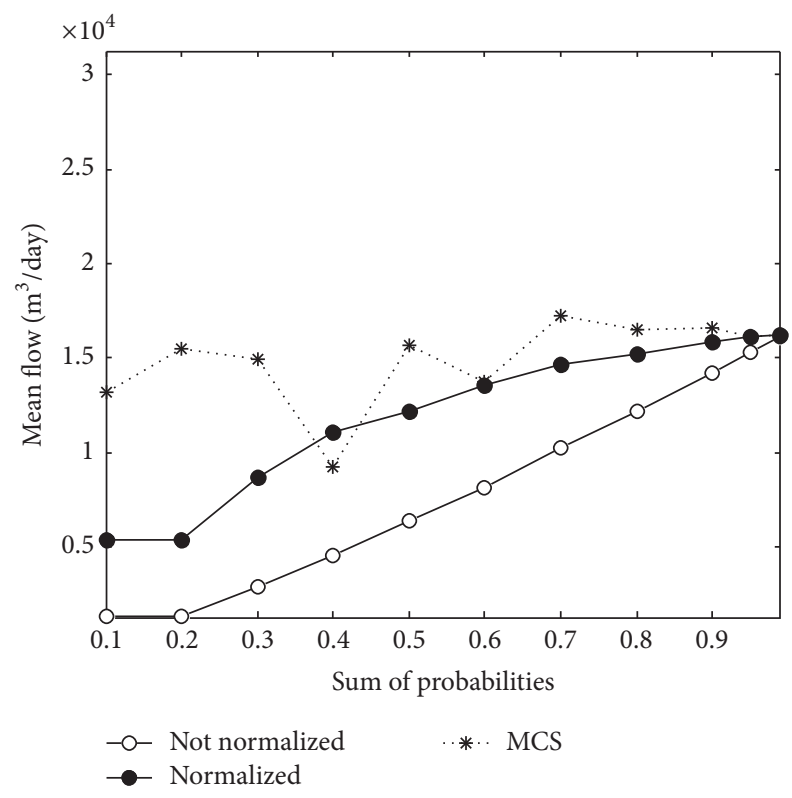

Figure 9: Comparison of results obtained with proposed branchand-bound approach and by Monte Carlo simulation for pipe 2 .

$9205.8 \mathrm{~m}^{3} /$ day, which is very close to that shown in Table 1 $\left(9164 \mathrm{~m}^{3} /\right.$ day). The line for the branch-and-bound method shows a flat line approximately from when the sum of the probabilities is 0.5 while the line for MCS shows constant fluctuations. This is because the branch-and-bound strictly sequences the system states and the associated quantities based on the order of the probabilities. On the other hand, MCS sequences the system states relatively loosely due to

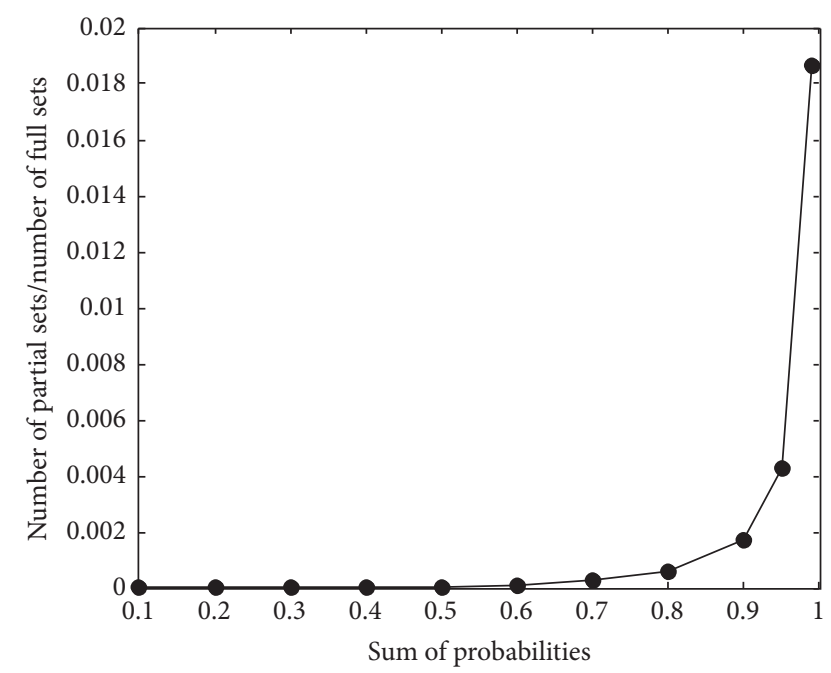

Figure 10: Number of identified branches in branch-and-bound analysis, normalized by number of full system states.

its random nature although it also sequences the system states based on the order of probabilities. In MCS, the probability for each system state is estimated based on the relative occurrence rates for random samplings, and MCS takes longer than the branch-and-bound approach, as the latter directly calculates the probability of each system state.

Figure 7 shows the results for pipe 6 , for which the overall trend is similar to that of the results for pipe 5. As the sum of the probabilities reaches 1 , the normalized results obtained with the branch-and-bound method exhibit good convergence to a value of $-2935.7 \mathrm{~m}^{3} /$ day, which is close to the value reported in Table $2\left(-2907.3 \mathrm{~m}^{3} /\right.$ day $)$. Meanwhile, the results obtained with MCS show continuous fluctuations.

Figures 8 and 9 show the results for pipes 1 and 2. The overall trend is again the same, but in Figure 9, the line for the branch-and-bound method continues to climb until the sum of the probabilities reaches 0.99 without any fluctuations. This differs from the other three figures and shows that even the branches with very low probabilities continue to increase the predicted flow. In this case, we cannot predict the flow based only on the branches identified in the branchand-bound analysis that have a sum of probabilities that is significantly lower than 1 , and further branching is needed for better convergence. This is mainly because the branchand-bound method sequences the system states based only on the order of the probabilities, although the predicted flow is calculated as the product of the probabilities and quantities. However, the sequencing of the system states based on both the probabilities and quantities is extremely time-consuming because the sequencing of quantities requires full evaluation of the hydraulic analysis algorithm for each branch. MCS still exhibits fluctuations even when the sum of probabilities is close to 1.

Figure 10 shows the number of partial sets identified during the branching process normalized by the number of 


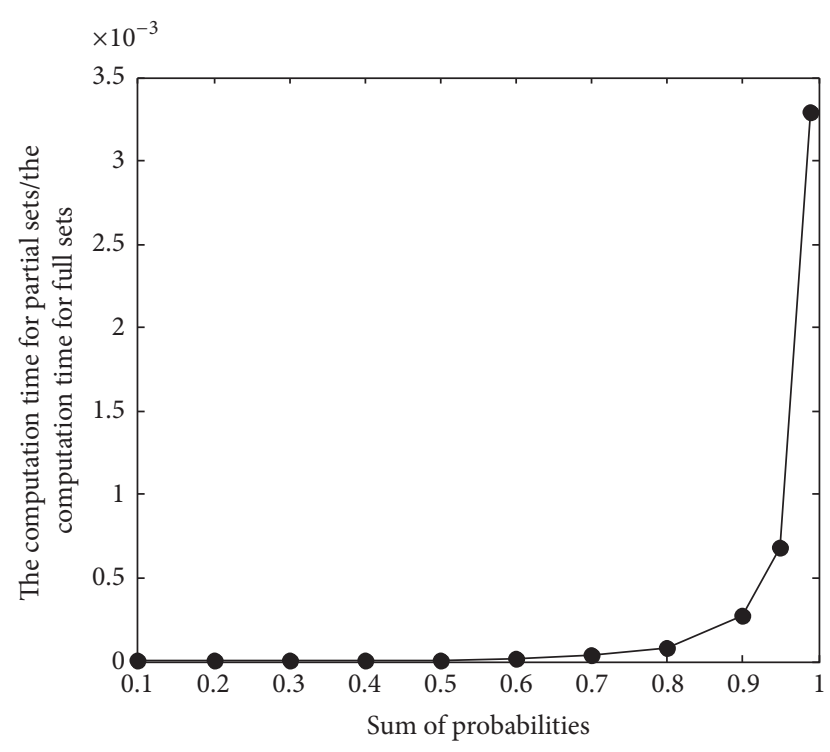

FIGURE 11: Computation time for branch-and-bound analysis normalized by computational time required to evaluate overall system state.

full sets in the entire system, with respect to the sum of the probabilities of the branches that reach a certain value. The figure shows that the required number of branches increases exponentially with the sum of the probabilities. This is reasonable because, as the sequencing in the branch-andbound analysis proceeds, the remaining branches assume smaller probabilities, and more time is needed to achieve the same value as the sum of the probabilities. However, even when the sum of the probabilities reaches 0.99 , the required number of branches in the branch-and-bound analysis is only $2 \%$ of the number of all the system states. This shows the significant computational saving of using the branch-andbound approach. When the sum of the probabilities is 0.99 , the branch-and-bound approach results have a $0.1 \%$ relative error in comparison with the results of considering all the sets of the system states.

Figure 11 shows the relative computation costs for the branch-and-bound analysis, relative to the cost required for the analysis of the full sets of the system states. This also shows an exponential increase in the computational costs, similar to the number of identified branches. The time to cost ratio when the sum of probabilities is 0.99 is only $0.33 \%$ of that of the full sets, which is even much lower than the ratio of the number of identified branches. This demonstrates the significant computational efficiency of using the branch-andbound approach.

Note that the branch-and-bound method in the proposed framework is based on the order of the probabilities of the system states without considering the system quantities, although we are interested in the prediction of flow rate quantities in terms of their partial descriptors which are the functions of both the probabilities and the associated quantities as shown in (3). Hence, the convergence of the branch-and-bound method may not be robust, and a sudden step change can occur, even after a large number of branches are identified, making it difficult to determine the truncation point of the branch-and-bound method. However, if the branching process is performed by considering both probabilities and quantities, it will significantly decelerate the computation because the order of the branches cannot be determined until each branch is fully expanded. To avoid sudden quantity changes during branching processes, any expected extreme quantities with a low probability should, if possible, be preidentified and then manually incorporated into the reliability analysis to avoid such convergence issues.

3.2. 20-Pipe Water Supply Network in Kobe City. The proposed framework was extensively applied to the 20-pipe water supply network shown in Figure 12 [21]. The network is part of a larger water supply system in Kobe, Japan, and consists of 120 service reservoirs, although water is supplied to the area shown in the figure from only one reservoir. This network was previously studied by Hoshiya et al. [21] to estimate the network redundancy. In this study, it was redigitized using the modeling procedure in the proposed framework. Figure 13 shows the modeling of the network in Google Earth, and Figure 14 shows the hydraulic model of the network in EPANET. Table 3 shows the link and node information including pipe material types, pipe segment lengths, the probability of damage to each pipe segment, and the water demand at the pipe junctions. Based on Javanbarg [22] and Zolfaghari and Niari [9], two pipe damage types, leaks and breaks, are categorized, and their probabilities are assigned as $80 \%$ and $20 \%$ for cast-iron pipes (CIP) and $100 \%$ and $0 \%$ for ductile iron pipes (DIP), assuming the occurrence of an earthquake identical to the one that struck Kobe in 1995. The probabilities of leaks from and breaks to a pipe are computed as a product of the following two probabilities: the failure probability in Table 3 and the probability of the occurrence of either a leak or break occurring when the pipe is damaged. The undamaged state is calculated as the probability of neither pipe leakage nor breakage occurring. In this example, we focus on the mean prediction of flows using the proposed branch-and-bound method procedure to check the applicability of the method to a larger network.

Figure 15 shows the predicted mean flows for pipes 4, 13, 16 , and 20 . The branching was carried out until the sum of the probabilities of the identified branches reaches 0.5. Further branching was not performed due to the high computational cost, given that 7.18 hours are needed to obtain a sum of probabilities of 0.5 with MATLAB on a computer with an Intel Core i7 CPU $930(2.80 \mathrm{GHz})$ and $3 \mathrm{~GB}$ of RAM. Further branching could be performed but the computation time would increase exponentially, which may not be affordable in most cases. However, these figures exhibit good convergence for the pipes considered in the figure, thus demonstrating the applicability of the branch-and-bound method to a large network. This applies for most of the pipes in the network. If we compare the number of existing total system states in the previous 11-segment network with that in the current 20segment network, the latter is 19,683 times the former. 


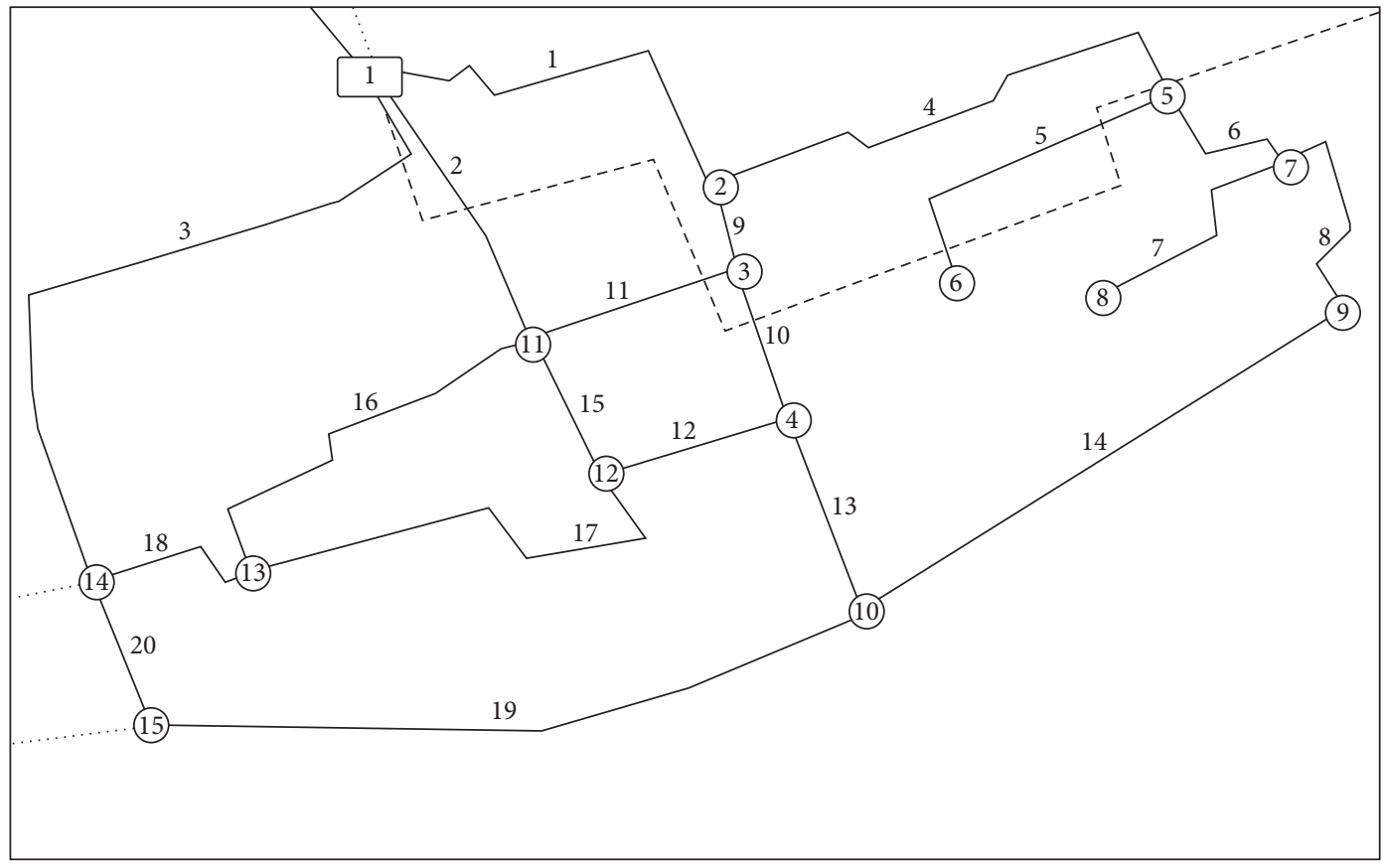

Supply node

Demand node Link
- - - New transmission pipe line

Connection pipe between neighboring areas

FIGURE 12: A 20-pipe water supply network in Kobe city.

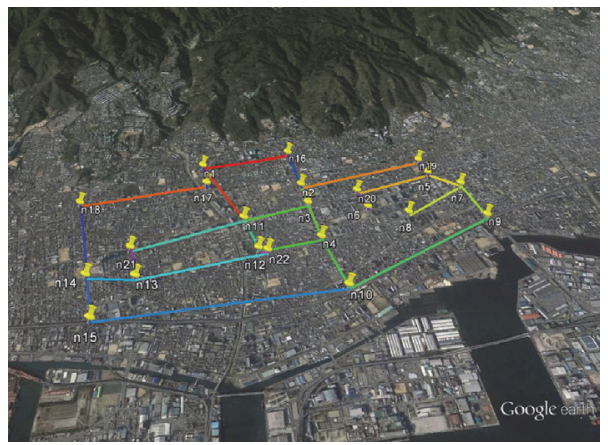

FIGURE 13: Modeling of Kobe city network in Google Earth.

However, Figure 16 shows the predicted mean flows for pipes $1,2,3$, and 9, which show not good convergence compared to the other pipes. Although the predicted flows in most of the pipe segments exhibit reasonable convergence like pipes $4,13,16$, and 20; the results of these four pipes exhibit a bit worse convergence, although they do not fluctuate but continue to increase or decrease with respect to the sum of the probabilities. These results suggest that further branching is needed or network simplification or other dimension reduction techniques need to be used to achieve the better convergence. However, this is a common difficulty of any currently existing network flow analysis methods, including MCS.

Figure 17 compares the number of identified branches with the number of full system states. When the sum of the probabilities is 0.5 , the relative number of identified branches is $0.00045 \%$, which is very small in comparison with the full system state evaluation. In the branch-and-bound approach, branching is carried out based on the sequencing of probabilities, and those system states with large probabilities are identified first. The system states with small probabilities are identified later. This means that, to achieve the same 


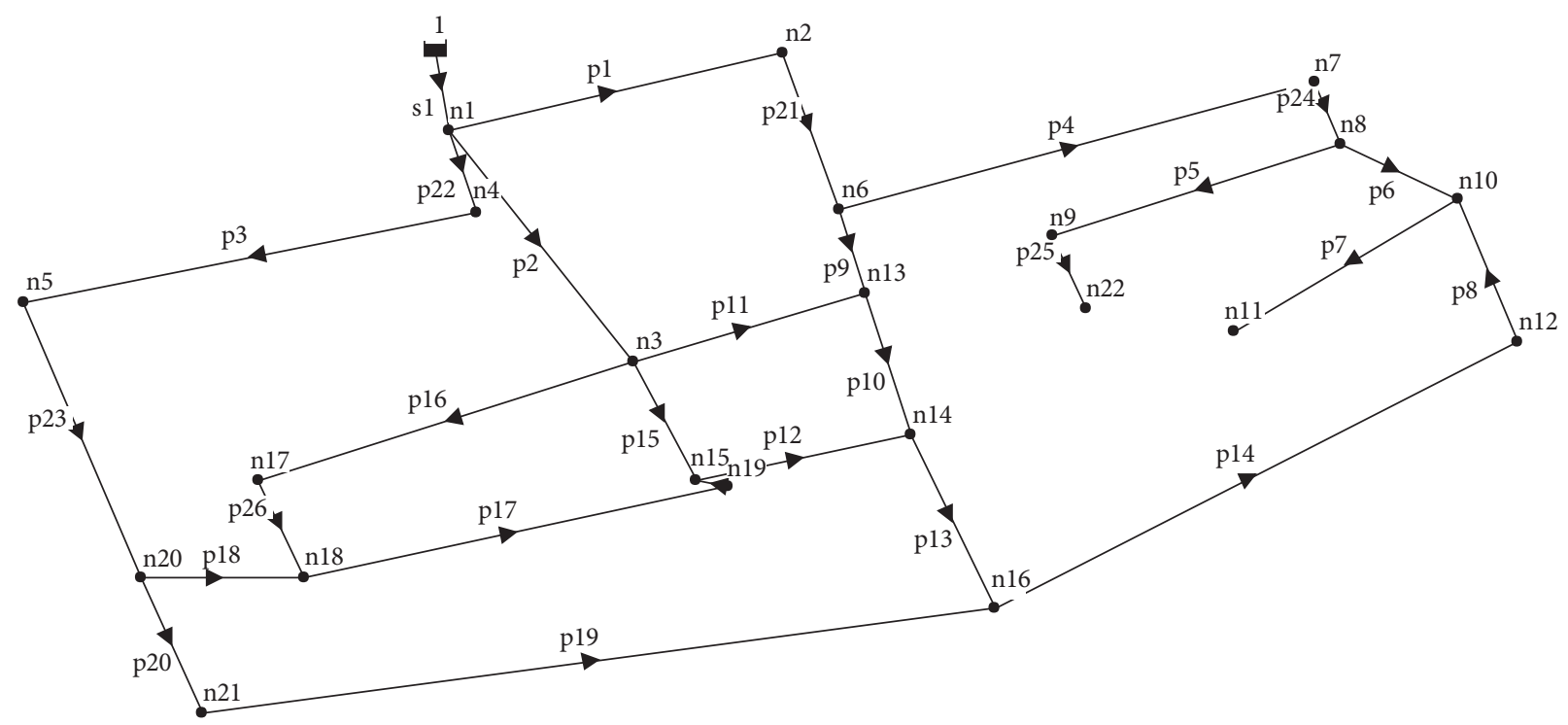

FIGURE 14: Hydraulic model of Kobe city network in EPANET.

TABLE 3: Link and demand node data of water distribution network [21, 22].

\begin{tabular}{|c|c|c|c|c|c|c|}
\hline Link number & Length $(\mathrm{km})$ & Pipe type & Diameter (mm) & Probability of failure & Node number & Water demand $\left(\mathrm{m}^{3} /\right.$ day $)$ \\
\hline 1 & 1.183 & CIP & 400 & 0.2844 & 2 & 2237 \\
\hline 2 & 0.818 & DIP & 400 & 0.2084 & 3 & 1678 \\
\hline 3 & 2.193 & DIP & 800 & 0.3474 & 4 & 3356 \\
\hline 4 & 1.472 & DIP & 400 & 0.5721 & 5 & 4475 \\
\hline 5 & 0.973 & CIP & 400 & 0.4211 & 6 & 2797 \\
\hline 6 & 0.474 & DIP & 400 & 0.2126 & 7 & 2237 \\
\hline 7 & 0.726 & DIP & 400 & 0.4049 & 8 & 3356 \\
\hline 8 & 0.673 & DIP & 400 & 0.2717 & 9 & 3356 \\
\hline 9 & 0.248 & CIP & 800 & 0.0855 & 10 & 5593 \\
\hline 10 & 0.440 & CIP & 800 & 0.1514 & 11 & 2237 \\
\hline 11 & 0.616 & CIP & 400 & 0.1751 & 12 & 5593 \\
\hline 12 & 0.522 & DIP & 400 & 0.1621 & 13 & 3915 \\
\hline 13 & 0.562 & CIP & 800 & 0.1677 & 14 & 3915 \\
\hline 14 & 1.559 & CIP & 400 & 0.3930 & 15 & 5034 \\
\hline 15 & 0.411 & DIP & 400 & 0.1155 & & \\
\hline 16 & 1.230 & DIP & 400 & 0.3099 & & \\
\hline 17 & 1.409 & DIP & 400 & 0.3429 & & \\
\hline 18 & 0.524 & CIP & 400 & 0.1492 & & \\
\hline 19 & 2.033 & CIP & 400 & 0.8942 & & \\
\hline 20 & 0.437 & DIP & 400 & 0.1490 & & \\
\hline
\end{tabular}

DIP: ductile iron pipe; CIP: cast-iron pipe.

probability as the previously identified branches, a greater number of branches is required. This is the reason for the exponential increase in the number of branches in the figure.

Figure 18 shows the measurement of exact computation time using MATLAB running on a computer with an Intel
Core i7 CPU $930(2.80 \mathrm{GHz})$ and $3 \mathrm{~GB}$ of RAM. In this network example, it is impossible to directly measure the computing time required for the analysis of all the branching due to the excessive computational cost required to carry out hydraulic analysis for $3.5 \times 109$. Instead, an extrapolated 


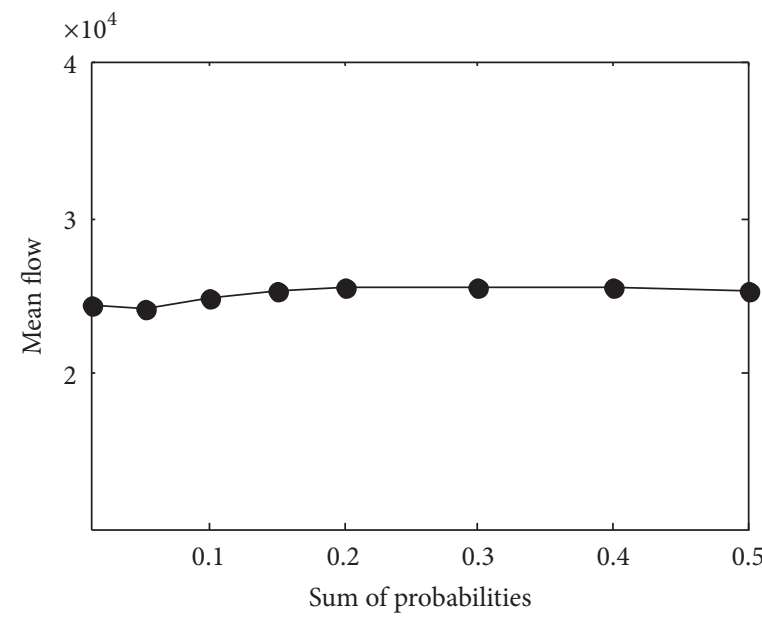

(a)

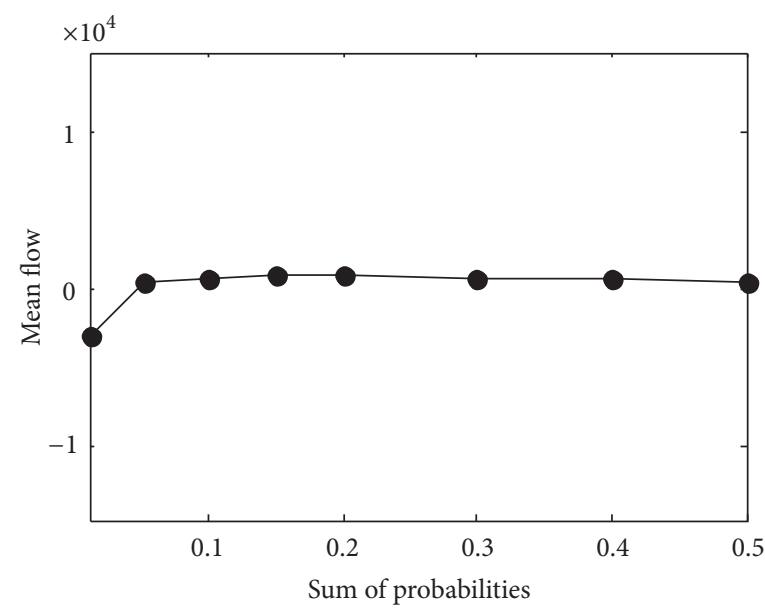

(c)

FIGURE 15: Predicted flows ( $\mathrm{m}^{3} /$ day) for pipes (a) 4, (b) 13, (c) 16, and (d) 20 using the branch-and-bound method.

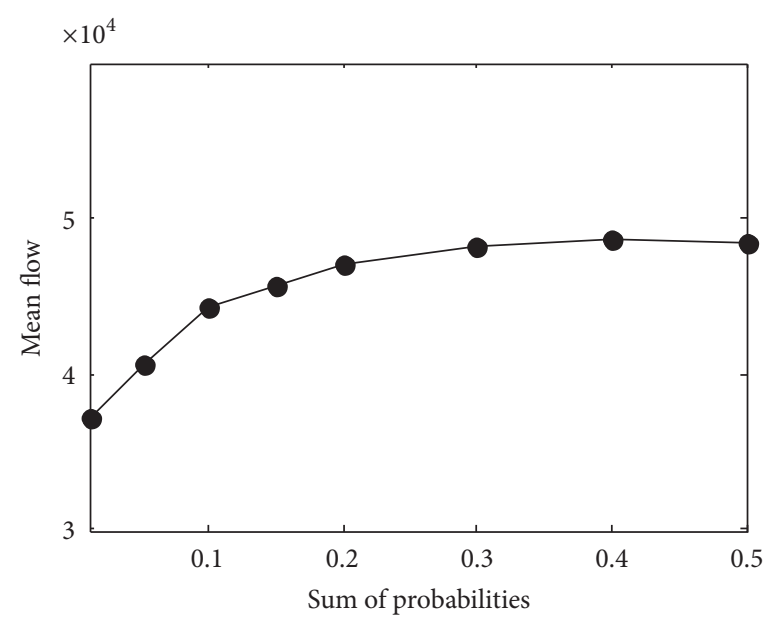

(b)

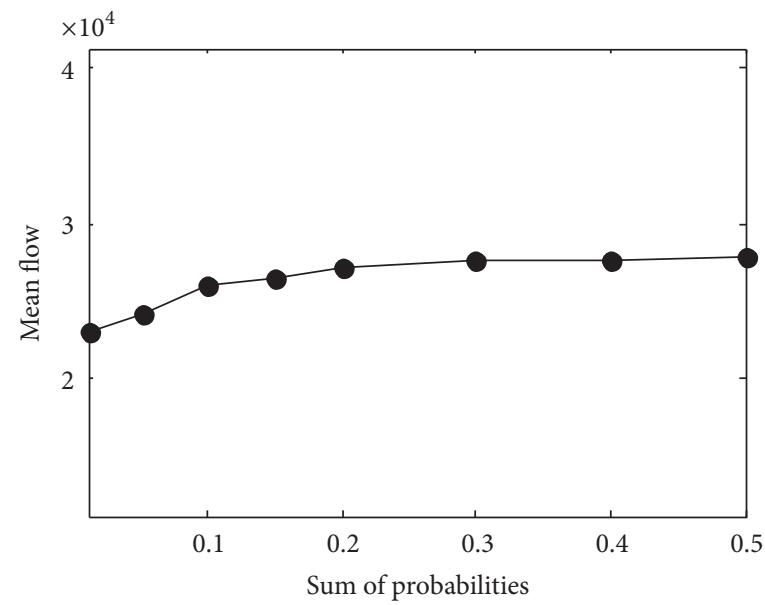

(d) approximation obtained by running a partial analysis estimates the required computing time as 23,212 days. This is obviously unaffordable relative to the time taken by the branch-and-bound analysis, that is, 7.18 hours.

\section{Conclusion}

This study has proposed an efficient system-level probabilistic flow analysis framework for estimating the postevent performance of a water pipe network in a probabilistic way. The framework consists of two parts: (1) a posthazard network modeling and (2) an efficient system-level probabilistic flow analysis algorithm developed based on the MSR method and the branch-and-bound method. The framework was demonstrated by application to two water pipe networks with 11 and 20 pipe segments, which experienced an earthquake event. For various seismic damage scenarios, the system performance was probabilistically measured in terms of the water flows in the network pipes. The results of the analysis showed that the flow rates in the pipes are determined to maintain the required demand at a specified node and that more damage to a pipe results in greater water loss. Computational efficiency was achieved by using the branchand-bound method and sequentially prioritizing the system states according to their likelihoods and considering only the important scenarios. This computational efficiency was demonstrated through examples, and the convergence issues in the branch-and-bound method were discussed.

\section{Conflicts of Interest}

The authors declare that there is no conflicts of interest regarding the publication of this paper.

\section{Acknowledgments}

The authors thank Ms. Seungyeon Ji and Mr. Abdul Mauzir of Western Sydney University for their contribution to discussions about and improvement of this manuscript. This 


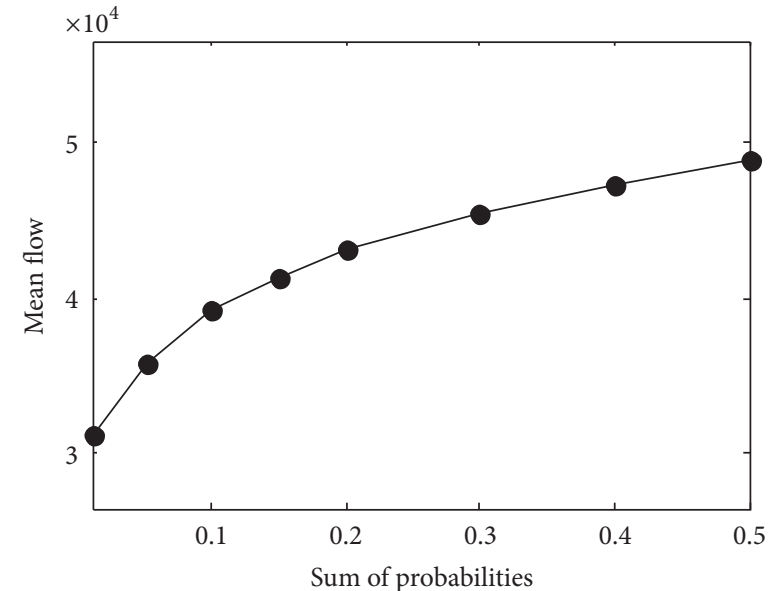

(a)

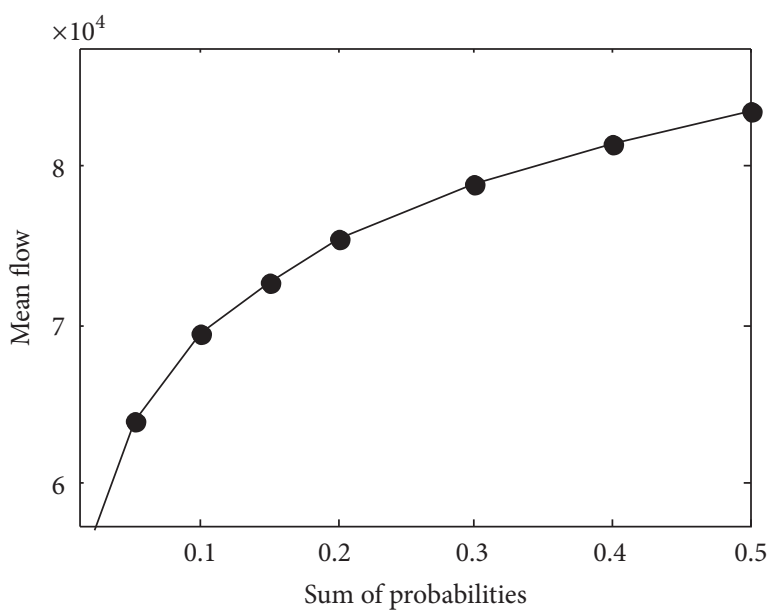

(c)

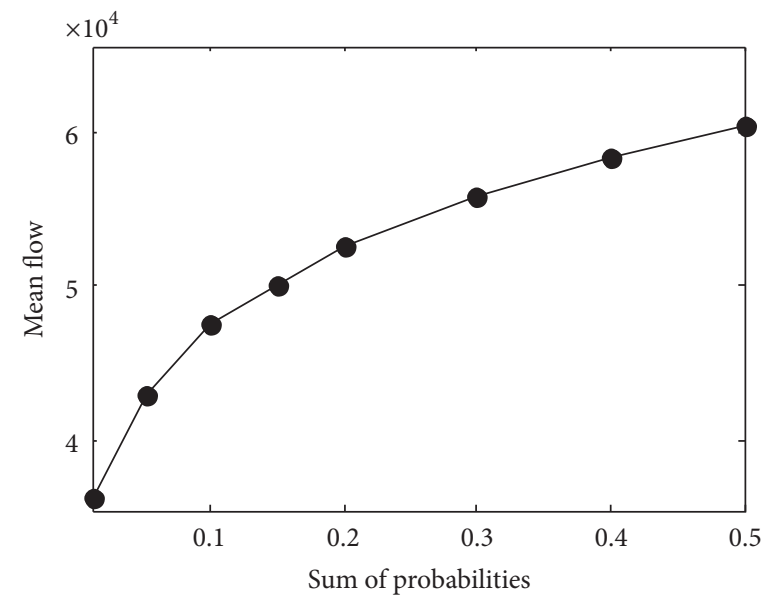

(b)

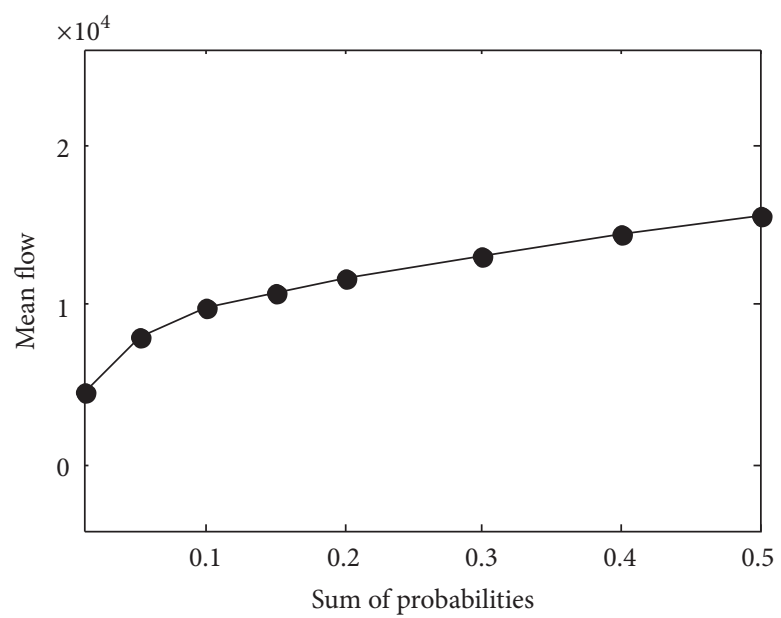

(d)

Figure 16: Predicted flows ( $\mathrm{m}^{3} /$ day) for pipes (a) 1, (b) 2, (c) 3, and (d) 9 using the branch-and-bound method.

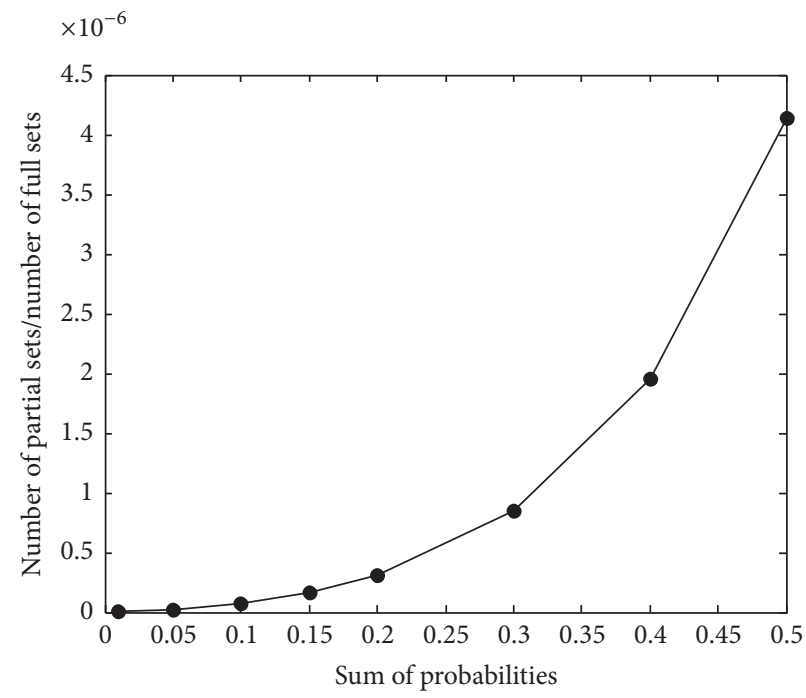

FIGURE 17: Number of identified branches in branch-and-bound analysis normalized by number of full system states. 


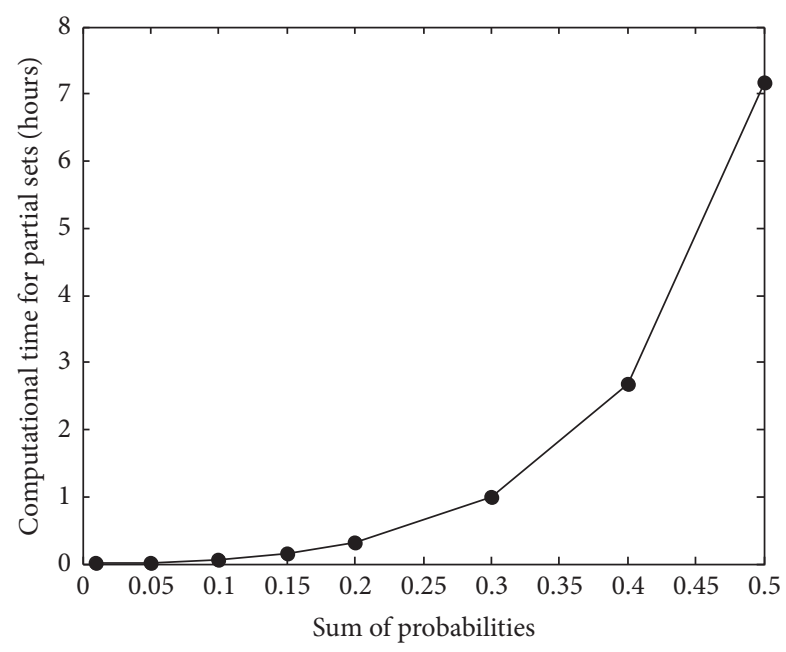

FIGURE 18: Computation time required for branch-and-bound analysis.

work was supported by the Australian Research Council (ARC) under its Linkage project (Project no. LP140100030). This work was also supported by the 2017 Research Fund (1.170013.01) of UNIST (Ulsan National Institute of Science and Technology).

\section{References}

[1] H. Alegre, Performance Indicators for Water Supply Services, IWA Publishing, 2006.

[2] L. Dueñas-Osorio, J. I. Craig, and B. J. Goodno, "Seismic response of critical interdependent networks," Earthquake Engineering and Structural Dynamics, vol. 36, no. 2, pp. 285-306, 2007.

[3] T. Adachi and B. R. Ellingwood, "Serviceability assessment of a municipal water system under spatially correlated seismic intensities," Computer-Aided Civil and Infrastructure Engineering, vol. 24, no. 4, pp. 237-248, 2009.

[4] Y. Kim, W.-H. Kang, and J. Song, "Assessment of seismic risk and importance measures of interdependent networks using a non simulation-based method," Journal of Earthquake Engineering, vol. 16, no. 6, pp. 777-794, 2012.

[5] I. Hernandez-Fajardo and L. Dueñas-Osorio, "Probabilistic study of cascading failures in complex interdependent lifeline systems," Reliability Engineering and System Safety, vol. 111, pp. 260-272, 2013.

[6] W.-H. Kang and A. Kliese, "A rapid reliability estimation method for directed acyclic lifeline networks with statistically dependent components," Reliability Engineering and System Safety, vol. 124, pp. 81-91, 2014.

[7] M. Fragiadakis, S. E. Christodoulou, and D. Vamvatsikos, "Reliability assessment of urban water distribution networks under seismic loads," Water Resources Management, vol. 27, no. 10, pp. 3739-3764, 2013.

[8] M. Fragiadakis and S. E. Christodoulou, "Seismic reliability assessment of urban water networks," Earthquake Engineering and Structural Dynamics, vol. 43, no. 3, pp. 357-374, 2014.

[9] M. R. Zolfaghari and M. A. Niari, "Probabilistic seismic damage assessment for water supply networks following earthquake," in Proceedings of the ASCE Technical Council on Lifeline Earthquake Engineering Conference (TCLEE '09), p. 119, Oakland, Calif, USA, July 2009.

[10] Y.-J. Lee, J. Song, P. Gardoni, and H.-W. Lim, "Post-hazard flow capacity of bridge transportation network considering structural deterioration of bridges," Structure and Infrastructure Engineering, vol. 7, no. 7-8, pp. 509-521, 2011.

[11] A. J. Torii and R. H. Lopez, "Reliability analysis of water distribution networks using the adaptive response surface approach," Journal of Hydraulic Engineering, vol. 138, no. 3, pp. 227-236, 2012.

[12] G.-Y. Liu, C.-H. Yeh, H.-Y. Hung, and K.-W. Chou, Seismic Analysis of Water Supply Systems by Earthquake Scenario Simulation, National Center for Research on Earthquake Engineering, 2012.

[13] Cornell University, GIRAFFE User's Manual, School of Civil and Environmental Engineering, Cornell University, Ithaca, NY, USA, 2008.

[14] D. G. Yoo, D. Jung, D. Kang, and J. H. Kim, "Seismic-reliabilitybased optimal layout of awater distribution network," Water, vol. 8, no. 2, article no. 50, 2016.

[15] ESRI, ArcGIS Desktop: Release 10, Environmental Systems Research Institute, Long Beach, Calif, USA, 2011.

[16] L. A. Rossman, EPANET 2: Users Manual, 2000.

[17] W.-H. Kang and J. Song, "Post-disaster damage detection for pipeline networks by matrix-based system reliability analysis," in Proceedings of the 11th International Conference on Applications of Statistics and Probability in Civil Engineering, p. 431, Zürich, Switzerland, August 2011.

[18] Y. Murotsu, H. Okada, K. Taguchi, M. Grimmelt, and M. Yonezawa, "Automatic generation of stochastically dominant failure modes of frame structures," Structural Safety, vol. 2, no. 1, pp. 17-25, 1984.

[19] Y. F. Guenard, Application of System Reliability Analysis to Offshore Structures, John A. Blume Earthquake Engineering Center, Stanford, Calif, USA, 1984.

[20] W.-H. Kang and Y.-J. Lee, "Efficient post-hazard probabilistic flow analysis of water pipe networks," in Proceedings of the 12th International Conference on Applications of Statistics and Probability in Civil Engineering (ICASP '12), Vancouver, Canada, July 2015.

[21] M. Hoshiya, K. Yamamoto, and H. Ohno, "Redundancy index of lifelines for mitigation measures against seismic risk," Probabilistic Engineering Mechanics, vol. 19, no. 3, pp. 205-210, 2004.

[22] M. B. Javanbarg, Integrated GIS-Based Seismic Performance Assessment of Water Supply Systems, Kobe University, Kobe, Japan, 2008.

[23] E. Todlini and S. Pilati, "A gradient method for the analysis of pipe networks," in Proceedings of the International Conference on Computer Applications for Water Supply and Distribution, Leicester, UK, 1987.

[24] D. Eliades, EPANET MATLAB Toolkit, 2009, http://www.mathworks.com/matlabcentral/fileexchange/25100-epanet-matlab-. toolkit.

[25] R. S. Pudar and J. A. Liggett, "Leaks in pipe networks," Journal of Hydraulic Engineering, vol. 118, no. 7, pp. 1031-1046, 1992.

[26] J. P. Vítkovský, A. R. Simpson, and M. F. Lambert, "Leak detection and calibration using transients and genetic algorithms," Journal of Water Resources Planning and Management, vol. 126, no. 4, pp. 262-265, 2000.

[27] Federal Emergency Management Agency (FEMA), HAZUSMHMR4 Technical Manual, FEMA, Washington, DC, USA, 2003. 
[28] M. O’Rourke and G. Ayala, "Pipelinpipeline damage due to wave propagatione damage due to wave propagation," Journal of Geotechnical Engineering, vol. 119, no. 9, pp. 1490-1498, 1993.

[29] R. Isoyama, E. Ishida, K. Yune, and T. Shirozu, "Seismic damage estimation procedure for water supply pipelines," Water Supply, vol. 18, no. 3, pp. 63-68, 2000.

[30] K. W. Campbell, "Empirical near-source attenuation relationships for horizontal and vertical components of peak ground acceleration, peak ground velocity, and pseudo-absolute acceleration response spectra," Seismological Research Letters, vol. 68, no. 1, pp. 154-179, 1997.

[31] W.-H. Kang, J. Song, and P. Gardoni, "Matrix-based system reliability method and applications to bridge networks," Reliability Engineering and System Safety, vol. 93, no. 11, pp. 1584-1593, 2008. 


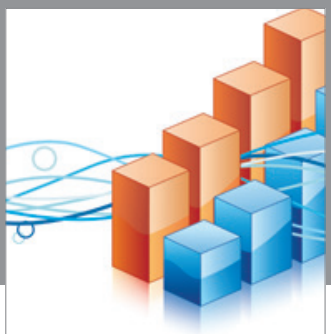

Advances in

Operations Research

vatem alat4

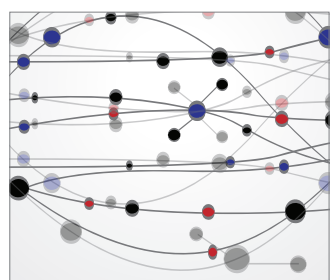

\section{The Scientific} World Journal
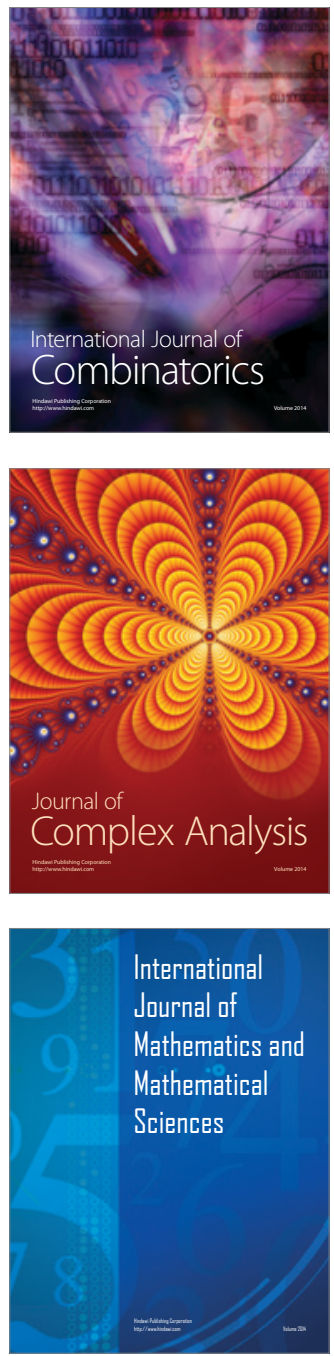
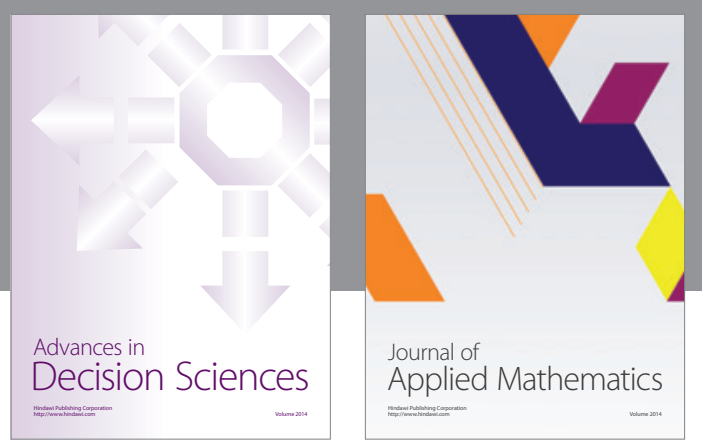

Algebra

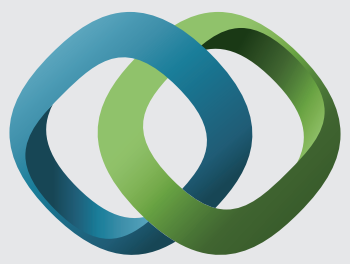

\section{Hindawi}

Submit your manuscripts at

https://www.hindawi.com
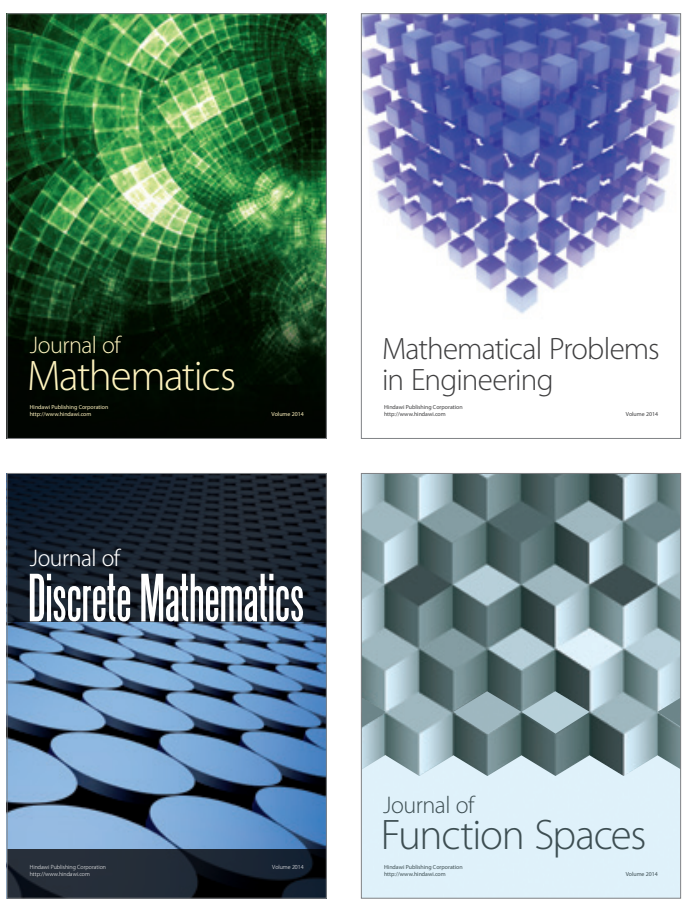

Mathematical Problems in Engineering
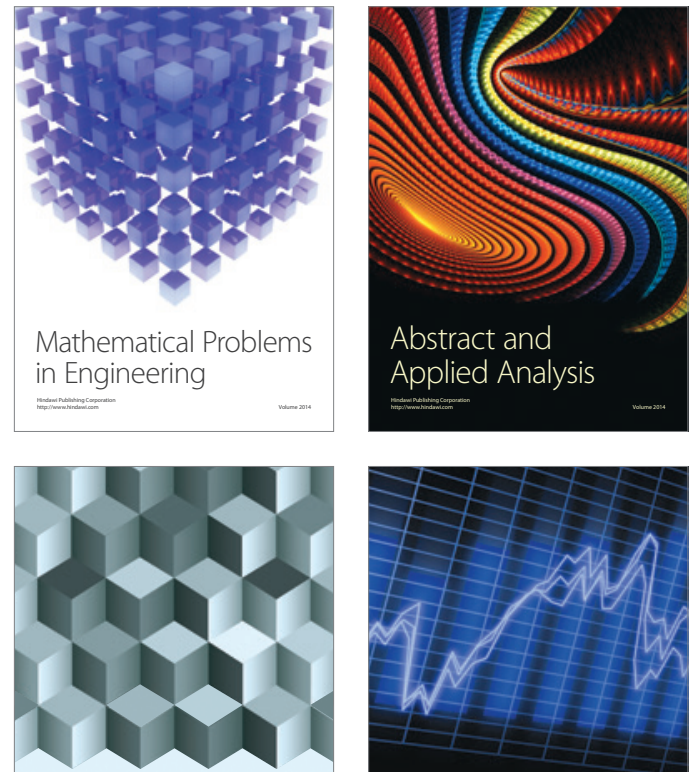

Journal of

Function Spaces

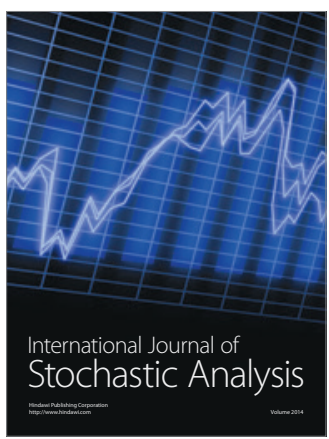

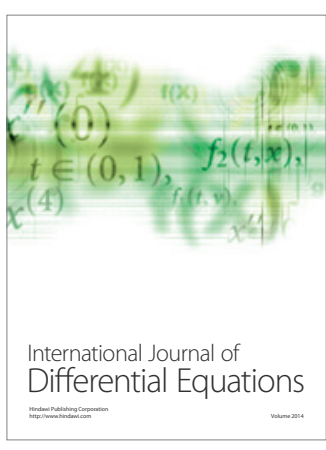
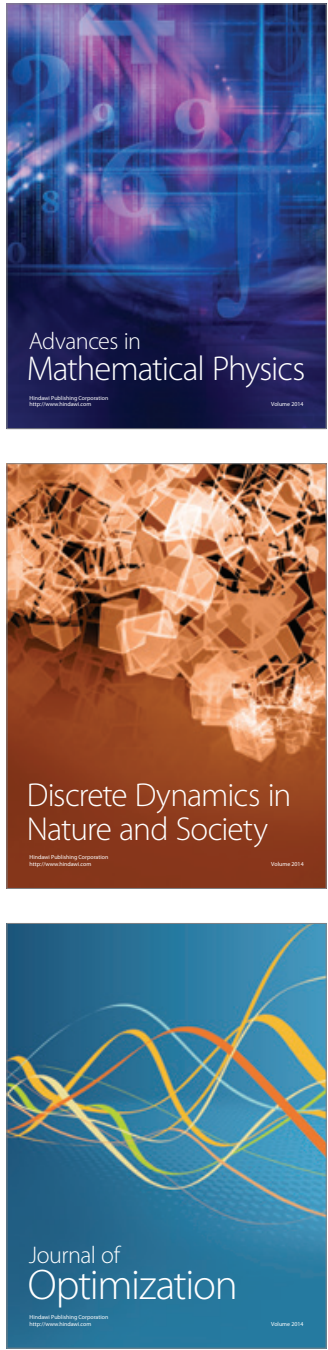